\title{
Laser interstitial thermal therapy of the spine: technical aspects
}

\author{
Nelson Moussazadeh, MD, ${ }^{1}$ Linton T. Evans, MD, ${ }^{1}$ Roxana Grasu, MD, ${ }^{2}$ Laurence D. Rhines, MD, ${ }^{1}$ \\ and Claudio E. Tatsui, MD'
}

Departments of ${ }^{1}$ Neurological Surgery and ${ }^{2}$ Anesthesiology, University of Texas MD Anderson Cancer Center, Houston, Texas

Spinal laser interstitial thermal therapy has been developed as a minimally invasive modality to treat epidural spinal tumors percutaneously. The safe and effective use of this technology requires meticulous preoperative trajectory planning and an intraoperative workflow incorporating navigation and MR thermography. Instrumented stabilization can be performed during the same operation if needed. Operative considerations and technical aspects are reviewed.

The video can be found here: https://youtu.be/P--frsag6gU.

KEYWORDS spinal laser interstitial thermal therapy; spinal metastasis 